\section{Vieillissement et rythmes biologiques chez les primates}

> Les rythmes biologiques constituent un avantage adaptatif puisqu'ils permettent d'harmoniser les rapports entre l'organisme et les variations cycliques de l'environnement. Avec l'âge, apparaissent des perturbations rythmiques caractérisées, le plus souvent, par des baisses d'amplitude et des décalages de phase. Hormis l'homme, les recherches sur les primates restent encore fragmentaires. Néanmoins, les données actuelles suggèrent que l'affaiblissement rythmique de l'organisme vieillissant procèderait d'un dysfonctionnement des noyaux suprachiasmatiques. Ces noyaux présentent des oscillations endogènes entraînées par la lumière et, avec l'âge, les capacités de réponses à la lumière seraient diminuées, se traduisant par une désynchronisation interne. La connaissance précise des altérations rythmiques liées à l'âge devrait suggérer, à terme, des traitements assurant le maintien ou la restauration de rythmes biologiques chez l'homme vieillissant, gage d'une meilleure qualité de vie et même de survie. <

Tous les organismes vivants présentent des activités rythmiques qui s'expriment depuis les bases moléculaires jusqu'aux fonctions physiologiques les plus intégrées. D’origine endogène, les rythmes biologiques sont synchronisés sur 24 heures par les variations cycliques de la lumière (alternance nuit/jour-durée du jour). Chez les mammifères, dont les primates et l'homme, les supports neuro-anatomiques et la chaîne fonctionnelle qui sous-tendent l'intégration du signal lumineux mettent au cœur du système les noyaux suprachiasmatiques (NSC), générateurs des rythmes endogènes et considérés comme le siège de l'horloge biologique. L'information lumineuse est transmise aux NSC dont l'activité est modulée par différents noyaux cérébraux et par la mélatonine. Produite uniquement la nuit par la glande pinéale, la sécrétion de mélatonine, par sa durée et son

Article reçu le 11 mai 2005, accepté le 16 septembre 2005

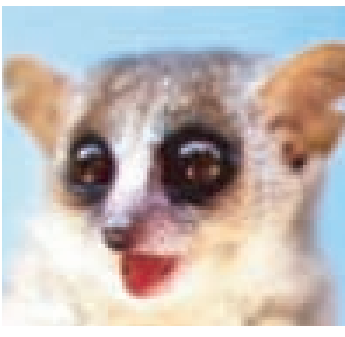

UMR 5176 (CNRS-MNHN), Département Écologie et gestion de la biodiversité, 4, avenue du petit Château, 91800 Brunoy, France. martine.perret@wanadoo.fr amplitude, détermine fabienne.aujard@wanadoo.fr un calendrier interne qui régule les rythmes journaliers et saisonniers des grandes fonctions physiologiques assurant l'homéostasie, la reproduction et la survie (Figure 1).

Les effets d'une désynchronisation des rythmes biologiques par rapport aux facteurs environnementaux restent peu connus mais, chez l'homme, les perturbations rythmiques pourraient être à l'origine des altérations physiques, métaboliques et pathologiques observées en situation de dérégulation temporelle artificielle (travail posté, voyages transméridiens), mais aussi lors du vieillissement. En effet, les études conduites chez les mammifères, y compris l'homme, ont démontré, qu'avec l'âge, apparaissaient des perturbations rythmiques caractérisées par des modifications d'amplitude, de phase ou de période selon les paramètres testés, modifications révélatrices d'une possible désynchronisation interne pouvant altérer la survie [1-5]. Ces effets du vieillissement ont surtout été démontrés sur les rythmes journaliers et peu de travaux concernent les rythmes saisonniers présents chez les primates et l'homme. Même si les facteurs socioculturels jouent un effet de masque, l'existence des rythmes saisonniers chez l'homme est attestée par divers paramètres parmi lesquels les rythmes de reproduction, les données épidémiologiques, les variations métaboliques et le statut hormonal sont les plus pertinents [6]. Ainsi, parmi toutes les théories actuelles sur le vieillissement [7], il a été suggéré que le déclin des performances physiologiques et comportementales observé lors du vieillissement, ainsi que l'apparition de pathologies, seraient en partie le résultat d'une désynchronisation des rythmes biologiques.

Chez les primates non humains, la connaissance des perturbations rythmiques au cours du vieillissement reste encore 
très fragmentaire et concerne essentiellement le macaque Rhésus et le microcèbe dont les longévités en captivité sont respectivement de 35-40 ans et 10-12 ans. Le microcèbe, un primate malgache (Figure 2), représente un support original pour les études sur le vieillissement du fait de ses rythmes adaptatifs de grande amplitude contrôlés par la photopériode $[8,9]$ et de ses pathologies liées au vieillissement très similaires à celles observées chez l'homme. Cette synthèse présente, de façon non exhaustive, les modifications des rythmes biologiques associées au vieillissement chez les primates, en référence à l'homme.

\section{Vieillissement et rythme d'activité générale}

Avec l'âge, l'activité générale et le rythme veille/sommeil s'altèrent chez l'homme. La fragmentation de ces rythmes s'explique en partie par une augmentation des

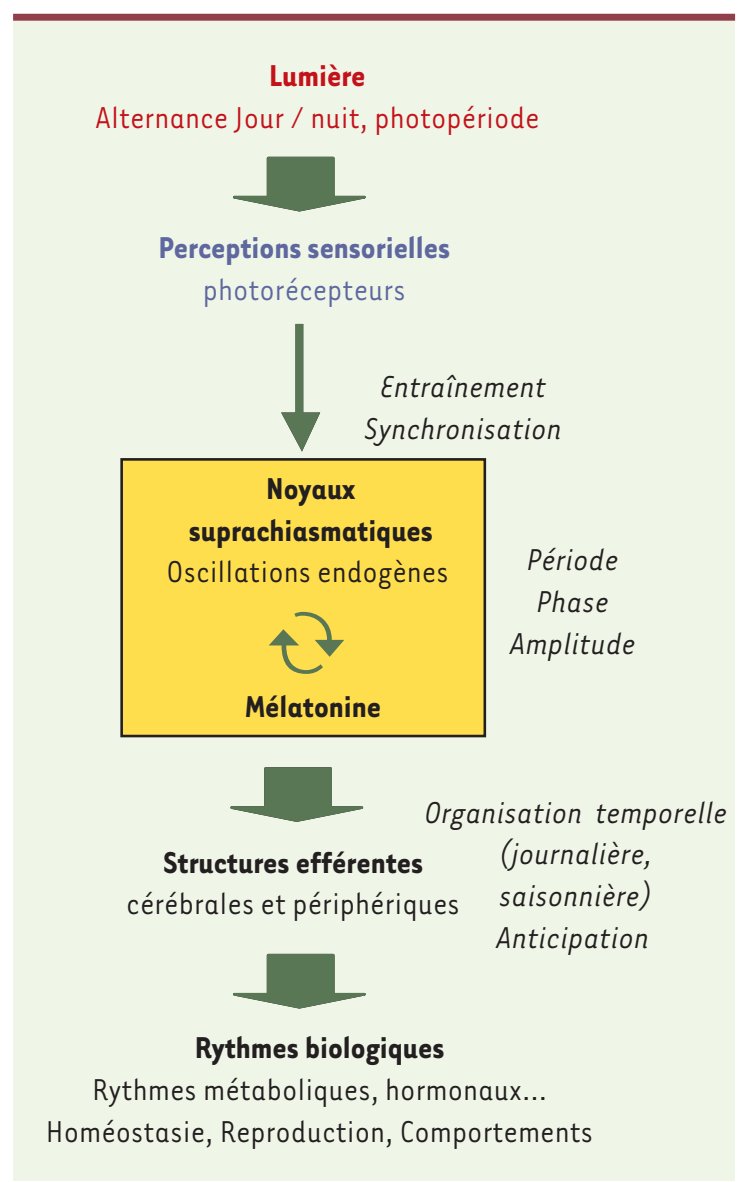

Figure 1. Schéma des structures impliquées dans l'expression des rythmes biologiques. Les oscillations endogènes des noyaux suprachiasmatiques et la sécrétion de mélatonine sont entraînées sur $24 \mathrm{~h}$ par l'alternance jour/nuit permettant la mise en place, au travers des structures centrales et périphériques, des rythmes journaliers et saisonniers. épisodes de sommeil pendant la journée et de fréquents réveils pendant la nuit $[1,10]$. À ces phénomènes, s'ajoutent des modifications dans les modalités de resynchronisation lors d'avances ou de retards de phase [11]. Chez les primates, peu d'études traitent du rythme veille/sommeil. Les rares indications existantes mentionnent une baisse de l'activité journalière chez le macaque [12]. Chez le microcèbe âgé (âge > 5 ans), l'activité locomotrice, strictement nocturne chez l'adulte, survient en avance de phase, se fragmente et des séquences d'activité sont présentes pendant le repos diurne (Figure 3). La resynchronisation des rythmes journaliers lors d'avance ou de retard de phase est également modifiée [13]. En outre, les résultats révèlent un dysfonctionnement des réponses à l'entraînement photopériodique, phénomène rarement mentionné dans les études sur le vieillissement des rythmes d'activité.

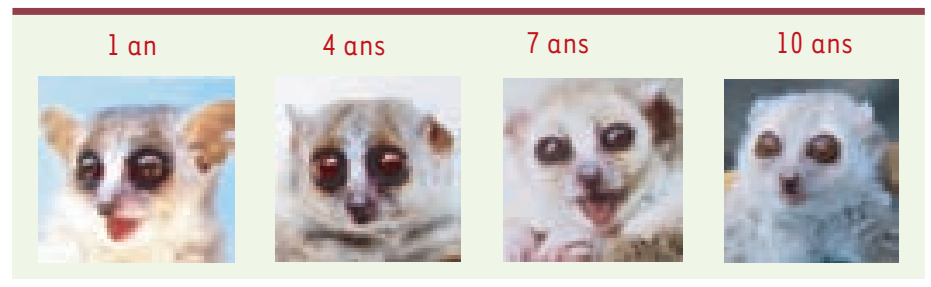

Figure 2. Évolution de la morphologie faciale en fonction de l'âge chez le Microcèbe.

\section{Vieillissement, température interne et métabolisme}

Avec l'âge, les profils de température interne chez l'homme, par nature en relation avec le rythme veille/sommeil, présentent une diminution de l'amplitude et s'accompagnent souvent d'une avance de phase du minimum matinal [10]. Chez le microcèbe, les perturbations du rythme et la baisse d'amplitude sont particulièrement marquées en raison de la modification de la phase de torpeur diurne présente chez cette espèce (Figure 3). En outre, les différences saisonnières dans l'expression de la torpeur diurne, normalement observées chez les animaux adultes, disparaissent chez les animaux âgés.

L'affaiblissement des rythmes de température interne est à mettre en parallèle avec les déficiences observées à différents niveaux du système de thermorégulation, associant baisse de thermogenèse et augmentation des pertes énergétiques au cours du vieillissement [10, 14]. Si chez les primates, les apports caloriques, la composition corporelle et les mécanismes qui les régulent évoluent avec l'âge, peu d'approches rythmiques y ont été consacrées. Chez le microcèbe, le métabolisme basal et la masse corporelle fluctuent en fonction des saisons de façon inverse avec de grandes amplitudes (Figure 4). Au cours du vieillissement, l'amplitude de ces variations diminue, reflétant un échappement au contrôle photopériodique des fonctions énergétiques. Les pertes énergétiques accrues au cours de la sénescence seraient, comme chez d'autres primates, liées à des modifications de la composition corporelle, des systèmes de thermorégulation et des processus d'acquisition de l'énergie. De nombreuses études chez l'homme âgé ont par ailleurs décrit tout un ensemble de modifications journalières dans les rythmes biochimiques, hématologiques ou immunologiques avec, le plus souvent, une baisse d'amplitude et des décalages de phase [2]. 
Parmi les hormones impliquées dans le déclin somatique au cours du vieillissement (synthèse des protéines, masse maigre, masse osseuse et fonction immunitaire), I'hormone de croissance (GH) présente, au cours du vieillissement et dans les deux sexes, une réduction des pulses nocturnes $[2,15]$. Les relations avec les modifications du rythme veille/sommeil restent à préciser. De façon identique, le vieillissement affecte la sécrétion de l'IGF-1 (insulin-like growth factor-1), un marqueur supposé de longévité lié au statut nutritionnel et métabolique [16]. Néanmoins, la composante rythmique de ces perturbations est encore à l'étude.

\section{Vieillissement et paramètres endocriniens}

\section{Axe hypothalamo-hypophyso-gonadique}

La diminution des fonctions de reproduction avec l'âge est un phénomène très étudié chez les primates. D’une façon générale, et dans les deux sexes, les rythmes journaliers des hormones sexuelles présents chez l'adulte s'affaiblissent ou disparaissent au cours du grand âge [2, 15, 17-20]. Pour le microcèbe, primate à reproduction saisonnière, les variations saisonnières de testostérone plasmatique persistent avec une grande amplitude jusqu'à un âge très avancé (6-7 ans) puis disparaissent. De même, la sécrétion d'œstrogènes diminue également avec l'âge chez la femelle microcèbe, mais sans effet direct sur la fécondité, cette espèce ne présentant pas de ménopause. À un niveau plus central, la baisse de l'activité gonadique s'accompagne d'une augmentation des hormones gonadotropes dont les rythmes journaliers disparaissent dans les deux sexes, mais avec persistance de rythmes saisonniers pour certaines d'entre elles [2, 21].

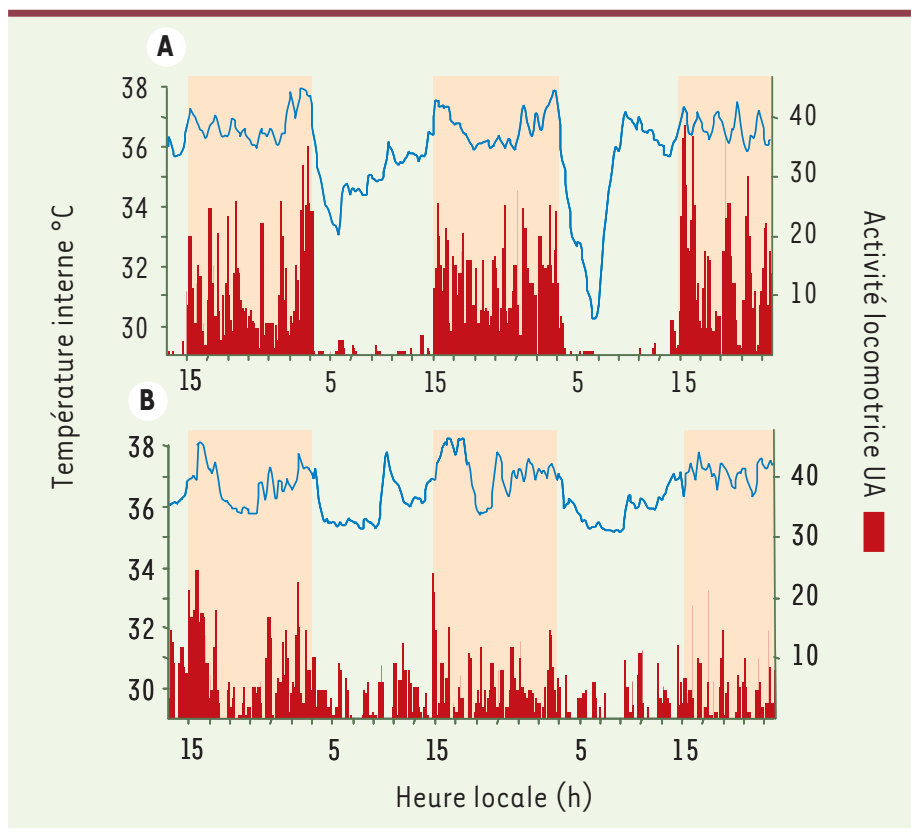

Figure 3. Profils journaliers de la température interne (courbe bleue) et de l'activité locomotrice (traits verticaux rouges) enregistrés chez le même microcèbe. A. Adulte, à l'âge de 3 ans. B. Adulte âgé, à l'âge de 7,5 ans. Le vieillissement s'accompagne d'une fragmentation et d'une baisse d'amplitude des rythmes. Les périodes nocturnes sont indiquées en jaune.

\section{Axe hypothalamo-hypophyso-thyroïdien}

Les variations journalières de la thyroxine, avec un pic en fin de journée, ne semblent pas affectées par le vieillissement [2]. Néanmoins, l'effet du vieillissement sur les variations saisonnières observées chez les primates n'est pas connu.

\section{Axe hypothalamo-hypo-surrénalien}

Parmi les hormones surrénaliennes, les variations journalières du cortisol ne semblent pas affectées par l'âge et la tendance à une avance de phase du pic matinal, pas toujours observée, serait dépendante des saisons [2, 20, 22, 23]. En revanche, la synthèse et production de déhydro-épiandrostérone (DHEA) et du sulfate de DHEA, phénomène apparemment spécifique aux primates, diminue au cours de l'âge chez l'homme dans les deux sexes et chez certains primates dont le microcèbe $[19,20,24]$. Considérés comme un marqueur du vieillissement biologique, les taux de DHEA seraient même indicateurs de la longévité intra- et interspécifique. Cette hormone présente un rythme journalier très accusé caractérisé par un pic en fin de matinée chez l'adulte, pic qui disparaît avec l'âge [22, 23].

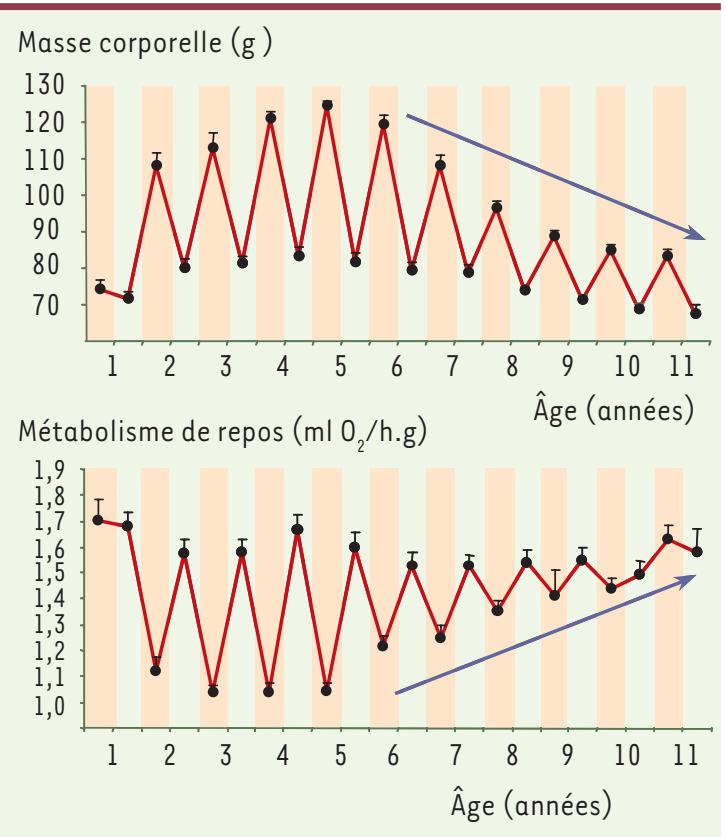

Figure 4. Évolution au cours de l'âge des variations saisonnières de la masse corporelle et du métabolisme de repos chez des microcèbes en captivité ( $\mu \pm s e m, n=16)$. En période hivernale (jaune), les animaux présentent une phase d'engraissement et une réduction métabolique. L'amplitude de ces variations saisonnières décroît après l'âge de la demi-vie ( 5 ans) suggérant une altération des réponses à la photopériode. 


\section{Effet du vieillissement sur les mécanismes centraux}

L'ensemble des données actuelles chez l'homme et les primates suggèrent que les perturbations rythmiques procèderaient d'une altération de l'entraînement par la lumière, ce qui sous entend une altération du fonctionnement des NSC dont l'origine peut être recherchée à différentes étapes d'intégration du signal lumineux. Le premier niveau concerne la réception de la lumière et s'il existe une opacification progressive du cristallin chez les primates âgés, son effet sur l'entraînement des rythmes reste à définir. En revanche, la sécrétion de mélatonine, qui reflète la transduction de la lumière, diminue avec souvent un décalage du pic nocturne chez les primates testés et l'homme $[5,20,25]$. Chez le microcèbe, les profils de mélatonine s'altèrent également avec le vieillissement et les différences saisonnières, normalement observées chez l'adulte jeune, s'estompent [26] (Figure 5A).

Les modifications du fonctionnement des NSC lors du vieillissement sont difficilement accessibles chez les primates. Par des études postmortem, une diminution du volume des NSC a été montrée chez l'homme âgé, accompagnée d'un déficit significatif de l'arginine-vasopressine (AVP) et/ou du polypeptide vaso-intestinal (VIP), neurotransmetteurs impliqués dans le fonctionnement rythmique des NSC [27]. Les rythmes

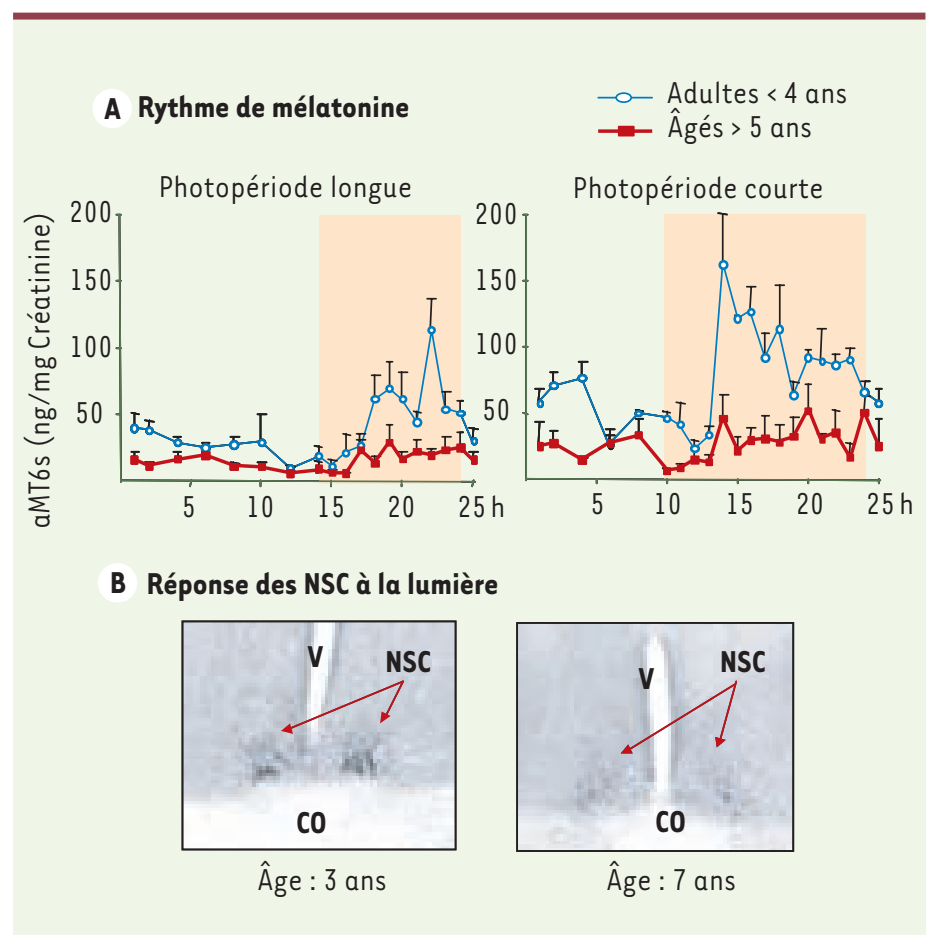

Figure 5. A. Profils journaliers de l'excrétion urinaire du principal métabolite de la mélatonine (aMT6s) chez des microcèbes jeunes et âgés $(\mu \pm S E M, n=12)$. Quelle que soit la photopériode (période nocturne en jaune), le rythme journalier disparaît chez les animaux âgés. $B$. Diminution de la réponse $c$-fos après un flash lumineux dans les noyaux suprachiasmatiques (NSC) de l'hypothalamus chez un microcèbe jeune et âgé. Ces deux paramètres indiquent un dysfonctionnement des réponses centrales à la lumière au cours du vieillissement. CO : chiasma optique; $\mathrm{V}: 3^{\mathrm{e}}$ ventricule [26]. journaliers et saisonniers de ces neurotransmetteurs sont également altérés avec l'âge, ce qui suggère une modification de l'activité fonctionnelle des NSC et de leur capacité à répondre à des signaux d'entraînement [28].

En absence de signaux d'entraînement journaliers (libre cours, désynchronisation forcée), la période endogène des NSC s'exprime. Cette période endogène, différente de 24 heures, semble se raccourcir chez l'homme, mais les données sont encore controversées tout comme chez les rongeurs. Chez le microcèbe, seul primate testé en longitudinal, la période endogène se raccourcit significativement avec l'âge. $\varepsilon$ n outre, chez le microcèbe, par une approche fonctionnelle des NSC, il a pu être montré que la réponse à un signal lumineux était abaissée chez les animaux âgés (Figure 5B) [26] et que les rythmes journaliers d'AVP et VIP, bien que présents, étaient en décalage de phase en comparaison avec des données chez l'adulte. Ces travaux rejoignent ceux effectués chez les rongeurs, démontrant à l'évidence que l'intégrité fonctionnelle des NSC est nécessaire au bon fonctionnement rythmique de l'organisme. En effet, l'implantation de NSC fœtaux sur des souris âgées permet une restauration des rythmes et un allongement de la survie [4]. Le vieillissement se reflète aussi dans l'expression des gènes de l'horloge [28, 29] avec des modifications de phase par rapport à l'entrâ̂nement lumineux, modifications susceptibles d'expliquer les désynchronisations rythmiques.

\section{Conclusions et perspectives}

Il est maintenant admis qu'il existe un avantage adaptatif de la rythmicité. Le maintien de rythmes biologiques synchronisés sur les facteurs d'entraînement est considéré comme un pronostic de longévité et un gage d'une meilleure qualité de vie. Compte tenu des recherches effectuées sur l'homme et les mammifères, des solutions ont été explorées pour améliorer les rythmes biologiques ou prévenir leur dégradation chez l'homme vieillissant. Diverses procédures ont été suggérées pour rétablir l'amplitude du rythme de température interne et supprimer l'avance de phase, avec des effets bénéfiques sur le sommeil. Parmi elles, l'exercice physique ou un bain chaud le soir permettent une augmentation de la dissipation de chaleur et favorisent ainsi la chute de température interne nécessaire à l'endormissement chez l'homme [10]. De même, l'exposition à une lumière forte ou une prise de mélatonine le soir permet de restaurer des profils de température interne. D'ailleurs, chez les rongeurs, l'apport de mélatonine aux animaux âgés restaure en partie les profils d'activité veille/sommeil et permet de meilleurs réponses à des décalages de phases.

Une grande voie en cours d'exploration est la supplémentation en hormones sexuelles ou en DHEA. Néanmoins, même si ces traitements apportent une meilleure qualité de vie, leurs 
effets sur l'expression des rythmes biologiques restent à étudier précisément. Enfin, une restriction calorique modérée au cours de la vie ralentirait les processus du vieillissement. Cette hypothèse, encore en cours de validation chez certains rongeurs et le macaque, pourrait s'accompagner du maintien de l'amplitude des rythmes biologiques [25, 30]. $\diamond$

\section{SUMMARY}

\section{Aging and biological rhythms in primates}

All living organisms exhibit rhythmic activities in a wide variety of endocrine and behavioural parameters. These biological rhythms are endogenously generated by a circadian clock, and they are entrained by cyclic variations of environmental factors called synchronizers. Aging is associated with changes in amplitude and temporal organization of many daily and seasonal rhythms. In humans, daily rhythms of sleep, thermoregulation and hormonal secretion are severely altered with aging. Except in humans, studies on primates are scarce. However, age-related effects on biological rhythms are relatively consistent among primate species studied to date, including humans. Therefore, non human primates are of valuable use for such investigations. Most studies have been performed on the Rhesus macaque (longevity 35-40 years) and on the gray mouse lemur (longevity 10-12 years). Like in humans, the restactivity rhythm becomes fragmented in aged primates, and shows an increased activity during the resting period. Aging induces a decrease in amplitude of the body temperature rhythm and an increase in energy consumption. Various hormonal secretions exhibit a decrease with aging, but the rhythmic components of these declines have not always been depicted. Moreover, changes (amplitude or phase) in daily variations depended of the hormonal secretion tested. Taken together, these results suggest that the biological clock in the brain would be a primary target of aging. The main central clock is located in the suprachiasmatic nucleus of the hypothalamus whose endogenous oscillations are entrained by light. In this brain structure, cellular function and sensitivity to light show drastic changes with age in the mouse lemur. The precise knowledge of agerelated alterations of biological rhythms in primates can have important consequences on the development of new treatments to maintain or restore biological rhythmicity in the elderly. $\diamond$

\section{RÉFÉRENCES}

1. Brock MA. Chronobiology and aging. J Am Geriatr Soc 1991; 39 : 74-91.

2. Touitou $Y$, Haus $\varepsilon$. Biologic rhythms in clinical and laboratory medicine. Berlin : Springer-Verlag, 1992: 730 p.

3. Perret M. Change in photoperiod cycle affects life span in a prosimian primate (Microcebus murinus). J Biol Rhythms 1997; 12 : 136-45.

4. Hurd MW, Ralph MR. The significance of circadian organization for longevity in the golden hamster. J Biol Rhythms 1998; 13: 430-6.
5. Touitou, Y. Human aging and melatonin. Clinical relevance. Exp Gerontol 2001 ; $36: 1083-100$.

6. Wehr TA, Moul DE, Barbato G, et al. Conservation of photoperiod-responsive mechanisms in humans. Am J Physiol 1993; 265 : R846-57.

7. Masoro EJ. Aging : current concepts. In : Schneider EL, Rowe JW eds. Handbook of the biology of aging. San Diego : Academic Press, $1996: 3-21$.

8. Perret M, Aujard F. Daily hypothermia and torpor in a tropical primate: synchronization by 24h light-dark cycle. Am J Physiol 2001 ; 281 : R1925-33.

9. Perret M, Aujard F. Regulation by photoperiod of seasonal changes in body mass and reproductive function in gray mouse lemurs (Microcebus murinus): differential responses by sex. Int J Primatol $2001 ; 22: 5-24$.

10. Van Someren EJ, Raymann RJ, Scherder EJ, et al. Circadian and age-related modulation of thermoreception and temperature regulation: mechanisms and functional implications. Ageing Res Rev $2002 ; 1: 721-78$.

11. Monk TH, Buysse DJ, Carrier J. Inducing jet-lag in older people : directional asymmetry. J Sleep Res $2000 ; 9: 101-16$.

12. Moscrip TD, Ingram DK, Lane MA, et al. Locomotor activity in female Rhesus monkeys: assessment of age and calorie restriction effects. J Gerontol $2000 ; 55:$ B373-80.

13. Cayetanot F, Van Sommeren EJW, Perret M, Aujard F. Shortened seasonal photoperiodic cycles accelerate aging of the diurnal and circadian locomotor activity rhythms in a primate. J Biol Rhythms $2005 ; 20: 461-9$.

14. Florez-Duquet M, McDonald RB. Cold-induced thermoregulation and biological aging. Physiol Rev $1998 ; 78: 339-58$.

15. Woller MJ, Everson-Binotto G, Nichols $\varepsilon$, et al. Aging-related changes in release of growth hormone and luteinizing hormone in female rhesus monkeys. J Clin Endocrinol Metab 2002 $87: 5160-7$.

16. Raynaud-Simon A, Lafont S, Berr C, et al. Plasma insulin-like growth factor 1 levels in the elderly: relation to plasma dehydroepiandrosterone sulfate levels, nutritional status, health and mortality. Gerontology $2001 ; 47$ : 198-206.

17. Chambers KC, Resko JA, Phoenix CH. Correlation of diurnal changes in hormones with sexual behavior and age in male Rhesus macaques. Neurobiol Aging $1982 ; 3: 37-42$.

18. Saal vom FS, Finch CE, Nelson JF. Natural history and mechanisms of reproductive aging in humans, laboratory rodents, and other selected vertebrates. In : Knobil $\varepsilon$, Neill JF eds. The physiology of reproduction. New York: Raven Press, 1994 : 1213-4.

19. Morley JE, Kaiser F, Raum WJ, et al. Potentially predictive and manipulable blood serum correlates of aging in the healthy human male: progressive decreases in bioavailable testosterone, dehydroepiandrosterone sulfate, and the ratio insulin-like growth factor 1 to growth hormone. Proc Natl Acad Sci USA 1997 ; 94 : 7537-42.

20. Goncharova ND, Lapin BA. Age-related endocrine dysfunction in nonhuman primate. Ann NY Acad Sci $2004 ; 1019$ : 321-5.

21. Shideler SE, Gee NA, Chen J, Lasley BL. Estrogen and progesterone metabolites and folliclestimulating hormone in the aged macaque female. Biol Reprod $2001 ; 65: 1718-25$.

22. Zhao ZY, Xie Y, Fu YR, et al. Circadian rhythm characteristics of serum cortisol and dehydroepiandrosterone sulfate in healthy Chinese men aged 30 to 60 years. A crosssectional study. Steroids $2003 ; 68: 133-8$.

23. Urbanski HF, Downs JL, Garyfallou VT, et al. Effect of caloric restriction on the 2-hour plasma DHEAS and cortisol profiles of young and old male rhesus macaques. Ann NY Acad Sci 2004 ; 1019: 443-7.

24. Labrie F, Bélanger A, Cusan L, et al. Marked decline in serum concentrations of adrenal C19 sex steroid precursors and conjugated androgen metabolites during aging. J Clin Endocrinol Metab 1997 ;

$82: 2396-402$.

25. Roth GS, Lesnikov V, Lesnikov M, et al. Dietaric restriction prevents the age-related decline in plasma melatonin levels of Rhesus monkeys. J Clin Endocrinol Metab $2001 ; 86: 3292-5$.

26. Aujard F, Dkhissi-Benyahya 0, Fournier I, et al. Artificially accelerated aging by shortened photoperiod alters early gene expression (Fos) in the suprachiasmatic nucleus and sulfatoxymelatonin excretion in a small primate, Microcebus murinus. Neuroscience 2001 ; $105:$ 403-12.

27. Hofman MA. The human circadian clock and aging. Chronobiol Int $2000 ; 17: 245-59$.

28. Yamazaki S, Straume $M$, Tei $H$, et al. Effects of aging on central and peripheral mammalian clocks. Proc Natl Acad Sci USA 2002 ; 99 : 10801-6.

29. Kolker DE, Fukuyama H, Huang DS. Aging alters circadian and light-induced expression of clock genes in golden hamsters. J Biol Rhythms 2003; 18 : 159-69.

30. Lane MA, Ingram DK, Roth GS. Nutritional modulation of aging in non human primates. J Nutr Health Aging $1997 ; 3: 69-76$. 